Research Paper

International Journal of Biological Sciences

ISSN 1449-2288 www.biolsci.org 2007 3(6):356-364

OIvyspring International Publisher. All rights reserved

\title{
The Amphioxus SoxB Family: Implications for the Evolution of Vertebrate Placodes
}

\author{
Daniel Meulemans and Marianne Bronner-Fraser
}

Division of Biology, Beckman Institute MC 139-74, California Institute of Technology, Pasadena, CA 91125, USA

Correspondence to: Dr. Daniel Meulemans, ph. 626395 3361, fax. 626449 8599, dm@caltech.edu

Received: 2007.05.21; Accepted: 2007.08.05; Published: 2007.08.06

Cranial placodes are regions of thickened ectoderm that give rise to sense organs and ganglia in the vertebrate head. Homologous structures are proposed to exist in urochordates, but have not been found in cephalochordates, suggesting the first chordates lacked placodes. SoxB genes are expressed in discrete subsets of vertebrate placodes. To investigate how placodes arose and diversified in the vertebrate lineage we isolated the complete set of SoxB genes from amphioxus and analyzed their expression in embryos and larvae. We find that while amphioxus possesses a single SoxB2 gene, it has three SoxB1 paralogs. Like vertebrate SoxB1 genes, one of these paralogs is expressed in non-neural ectoderm destined to give rise to sensory cells. When considered in the context of other amphioxus placode marker orthologs, amphioxus SoxB1 expression suggests a diversity of sensory cell types utilizing distinct placode-type gene programs was present in the first chordates. Our data supports a model for placode evolution and diversification whereby the full complement of vertebrate placodes evolved by serial recruitment of distinct sensory cell specification programs to anterior pre-placodal ectoderm.

Key words: Evolution, development, chordates, vertebrates, placodes, amphioxus

\section{INTRODUCTION}

The evolution of active predation in the vertebrate lineage was facilitated by the appearance of anterior paired sense organs and ganglia. Developmentally, these structures are derived from tissues considered unique to vertebrate embryos, the neural crest and cranial placodes. However, recent evidence of putative placode homologs in urochordates[1, 2], and new molecular phylogenies positioning this subphylum as the vertebrate sister group [3, 4], have cast doubt on the status of placodes as vertebrate synapomorphies. Gene expression in the tunicate Ciona and the larvacean Oikopleura suggest that the ancestor of vertebrates and urochordates had one or two composite placodes homologous to the adenohypophyseal, olfactory, and otic. The full set of vertebrate placodes is thought to have arisen by partitioning of these ancestral placodes. The possible existence of proto-placodes in the prevertebrate chordate effectively pushes back the origins of placodes to before the urochordate/vertebrate split. In this context, the most relevant outgroup for understanding placode origins becomes the cephalochordata, the third chordate subphylum.

Regardless of phylogenetic position, the cephalochordates are widely accepted as possessing the most prototypically chordate body plan of the three subphyla[5-7]. The likely basal position of cephalochordates reinforces this view and the utility of cephalochordates as proxies for the ancestor of both urochordates and vertebrates. To date, there is no strong evidence for the existence of placodes in amphioxus except for the possible homology of the preoral pit to the vertebrate adenohypophyseal placode[8-11]. Unlike urochordates and vertebrates, no region of amphioxus ectoderm appears to combine generic placodal properties such as localized thickening, invagination, and delamination with sense organ formation. However, amphioxus, like other invertebrate deuterostomes, does possess an epidermal nerve plexus including individual sensory neurons. The relationship of this plexus to vertebrate placodes is unclear. Gans and Northcutt originally proposed that placodes and neural crest evolved by reorganization of such a primitive plexus [12, 13]. Northcutt later rejected this in favor of replacement of the plexus by a non-homologous system derived from neural crest and placodes[14], as suggested by Lacalli[15]. While likely non-homologous at the tissue level, Lacalli left open the possibility of cell-level homology between components of the amphioxus and vertebrate peripheral nervous systems[15].

To further investigate possible cell or gene network-level homologies between placodes and components of the amphioxus epidermal nerve plexus we examined the expression of $\operatorname{SoxB}$ genes in amphioxus. The SoxB family consists of SoxB1 genes (vertebrate Sox1,2,3) and SoxB2 genes (Sox14,21)[16]. Both SoxB1 and SoxB2 genes are expressed broadly in the central nervous system (CNS) where they interact to drive neural induction and differentiation[17-19]. In the nascent peripheral nervous system (PNS), SoxB1 genes mark a subset of cranial placodes [20-23] and 
ectopic Sox3 is sufficient to induce supernumerary lens and otic vesicles [24]. SoxB1 genes also control the final stages of sense organ differentiation, directly activating delta-crystallin expression in the embryonic lens [25].

Here we report the isolation and expression of the complete set of SoxB genes from amphioxus. We find that like vertebrates, amphioxus has three SoxB1 genes, but like invertebrates, only possesses a single SoxB2 gene. Phylogenetic and genomic analyses and gene expression suggest that amphioxus and vertebrate SoxB1 paralogs arose via independent duplication events. In addition, we observe expression of an amphioxus SoxB1 co-ortholog in two populations of putative epidermal sensory cells. In the context of placode marker homolog expression our data suggests that discrete subpopulations of sensory cells utilizing placode-type genetic programs predate the evolution of placodes. Taken together, these data support a model for placodal diversification via repeated recruitment of preexisting sensory cell programs to an anterior placodal primordium.

\section{MATERIALS AND METHODS}

\section{Amphioxus Collection}

Amphioxus adults (Branchiostoma floridae) were collected from Tampa Bay, Florida and electrostimulated to induce gamete release. Eggs were fertilized, and embryos were cultured and fixed per the methods of Holland et al. [26].

\section{Isolation of amphioxus $\operatorname{Sox} B$ genes}

The following completely degenerate primers were designed against the HMG box of vertebrate Sox E proteins: AAGCCBCAYGTIAARMGNCCIATGAA, and TAITCIGGGTRRTCYTTYTTRTGYTG. Using these primers, a $220 \mathrm{bp}$ DNA fragment with high homology to vertebrate SoxB2 genes was amplified by PCR from an amphioxus Lambda Zap II embryonic cDNA library (a gift from Jim Langeland). This fragment was then used to screen the plated library at low stringency $\left(2 \mathrm{XSSC} / .1 \% \mathrm{SDS}\right.$ at $\left.40^{\circ} \mathrm{C}\right)$ for full-length cDNAs. Fourteen phagemid clones were isolated, excised, partially sequenced, and found to encode two different $\operatorname{SoxB}$ genes. The largest cDNAs of each were completely sequenced. Amphioxus SoxB1 and SoxB2 coding sequences were then used to search Amphioxus Genome Release v1.0 (Joint Genome Institute) for paralogs. An EST clone (clone ID bfne072e07) corresponding to amphioxus SoxB1b (gene model estExt_fgenesh2_pg.C_6920002) was obtained and sequenced (a gift from Jr Kai Yu).

\section{Phylogenetic analysis}

Full-length cDNA sequences were translated and their conceptual protein products were aligned to published sea urchin, ascidian, amphioxus, and vertebrate Sox group B sequences using ClustalX [27]. The Sox group E gene, chicken Sox8, was included as an outgroup. A bootstrapped Neighbor-Joining tree [28] and was then constructed using ClustalX, and drawn using NJplot [29]. Bootstrap values were calculated from 1000 resamplings of the alignment data. Maximum Likelihood trees and quartet-puzzling reliability scores were generated from the same alignment using TREE-PUZZLE [30]. Similar analyses were performed on truncated protein sequences encompassing only the conserved HMG domain.

\section{In situ Hybridization}

In situ hybridizations were as described previously [31]. Riboprobes were made against the entire transcript.

\section{RESULTS AND DISCUSSION}

\section{The SoxB family in amphioxus}

Using degenerate PCR and low-stringency library screening, we isolated two novel SoxB genes from amphioxus, one most similar to vertebrate SoxB1 genes, the other to vertebrate SoxB2 homologs. These genes were used to search Amphioxus Genome Release v1.0 for potential amphioxus paralogs. Two other putative SoxB1 genes were identified. The first corresponded to the previously described AmphiSox1/2/3[32]. The second was a previously undescribed gene with high similarity to AmphiSox $1 / 2 / 3$ located 9337bp downstream of AmphiSox1/2/3 on the same genomic sequence scaffold. Due to sequence similarity and genomic context, this gene likely represents a recent amphioxus duplicate of AmphiSox1/2/3. Based on order of discovery, we propose renaming AmphiSox1/2/3 as amphioxus SoxB1a and designating its most recent duplicate SoxB1b. The remaining amphioxus group B1 paralog is designated SoxB1c.

Bootstrapped Neighbor-Joining and Maximum Likelihood analyses group amphioxus SoxB1c and SoxB2 with their respective vertebrate homologs at high confidence values (Figure 1A). In contrast, placement of amphioxus SoxB1a and SoxB1b within the SoxB1 subgroup was only weakly supported by Neighbor-Joining and unsupported by Maximum Likelihood, despite high similarity to metazoan SoxB1 genes when queried against GenBank. Similarly, a single Ciona SoxB1 homolog failed to group with any other deuterostome SoxB sequences despite a clear affinity to SoxB1 subgroup members. Phylogenies using only the conserved HMG domains gave similar results (data not shown), suggesting amphioxus SoxB1a and SoxB1b and Ciona SoxB1 are fast-evolving members of the SoxB1 subgroup.

The failure of the three amphioxus SoxB1 genes to group with any single vertebrate SoxB1 paralog, and the presence of single SoxB1 homologs in sea urchin and Ciona, suggests that amphioxus SoxB1 genes arose by two lineage-specific duplication events. As indicated above, the similarity and genomic proximity of amphioxus SoxB1a and SoxB1b indicate they are the products of the most recent of these duplications, whereas SoxB1c arose during the first duplication. Several examples of similar amphioxus-specific gene duplications have been reported[33]. 

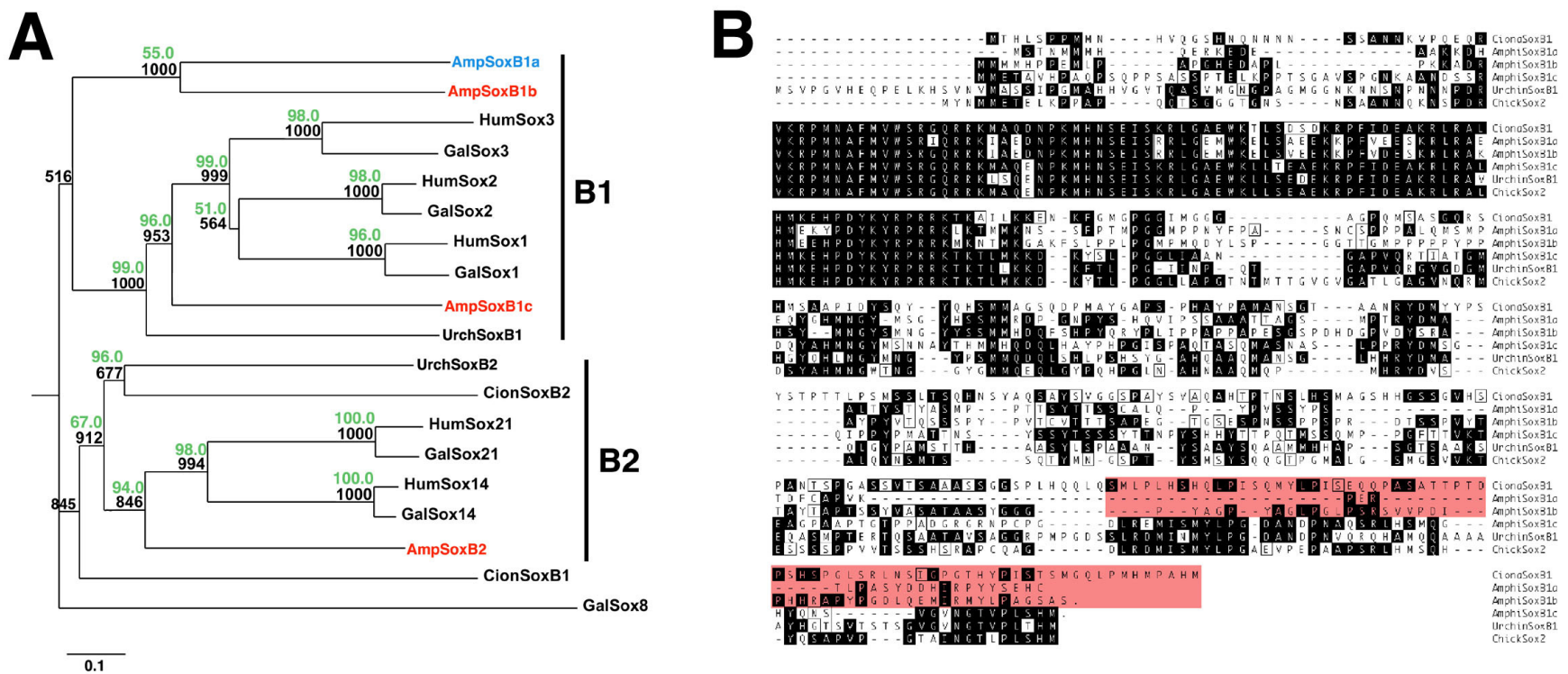

Figure 1. Phylogenetic tree and alignment of deuterostome SoxB proteins. (A) Phylogenetic tree created using the Neighbor-Joining method with chicken Sox8 as the outgroup. Black numbers at branch bases are confidence values derived from 1000 bootstrap resamplings of the alignment data. Sequence distance is indicated at the bottom left as substitutions per base. Numbers in green are quartet-puzzling reliability scores from Maximum Likelihood analysis of the same alignment. Amphioxus SoxB1c and amphioxus SoxB2 group with their respective vertebrate homologs. Amphioxus SoxB1a and SoxB1b group together outside a clade including SoxB1c and vertebrate and sea urchin SoxB1 genes. In both analyses, Ciona SoxB1 failed to group with the other SoxB1 genes. (B) Alignment of amphioxus, urchin, Ciona and chick SoxB1 proteins. While all proteins are highly conserved in N-terminal HMG domain, the C-terminal transactivation domain is only conserved in chick Sox2, urchin SoxB1 and amphioxus SoxB1c. Amphioxus SoxB1a, SoxB1b and Ciona SoxB1 all have divergent transactivation domains, suggesting a similar loss of functionality.

\section{Conserved roles for amphioxus $S o x B$ genes in the CNS and gut}

SoxB genes are required for the earliest steps of CNS formation in vertebrates [17] and Drosophila [34-36]. Consistent with an ancient role for SoxB1 genes in neurectoderm specification, amphioxus SoxB1 genes are all expressed in the nascent neural plate (Figure 2, Figure 3A-G) [32]. After neural induction and neurulation, vertebrate SoxB1 genes also act to maintain progenitor cell identity in the developing CNS. Like its vertebrate cognates, amphioxus SoxB1c persists in the neural tube until larval stages (Figure $3 \mathrm{H})$ suggestive of a conserved role in regulating neuronal differentiation. Like its vertebrate and Drosophila counterparts, amphioxus SoxB2 is coexpressed with SoxB1 paralogs in the developing CNS-- likely reflecting conserved interactions between these genes (Figure 4 ).

In vertebrates, SoxB1 genes are expressed in the foregut and posterior-most hindgut [37-40]. Similar SoxB1c expression is seen in the foregut and hindgut of amphioxus, suggesting a highly conserved function in patterning the chordate endoderm (Figure 3, all panels). Low levels of amphioxus SoxB2 transcripts are also detected in the endoderm at neurula stages (Figure 4, all panels). No similar expression is reported for vertebrate SoxB2 genes, though the Drosophila SoxB2 homolog, dichaete, is necessary for hindgut
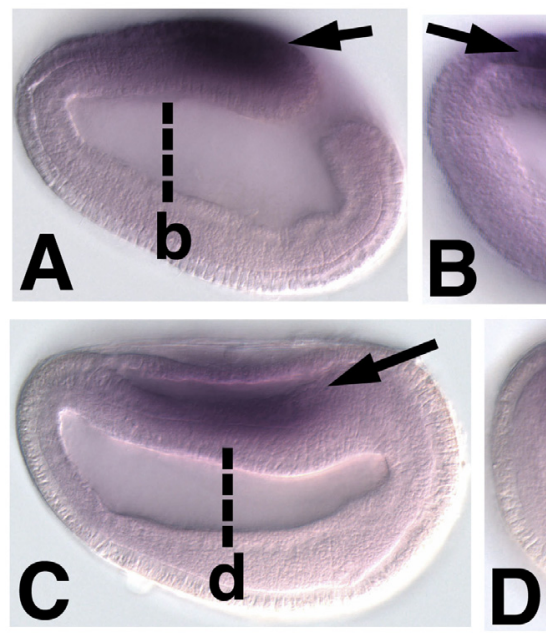

Figure 2. Embryonic expression of amphioxus SoxB1b. Anterior is to the left (A) Side view of 9-hour early neurula. SoxB $1 b$ expression is seen throughout the neural plate (arrow). (B) Optical cross section through 9-hour early neurula at approximately the level of $b$ in A (arrow). (C) Side view of 12-hour neurula. SoxB1b is expressed in the neural plate as it rolls-up to form the neural tube (arrow). (D) Optical cross section through 12-hour neurula at the level of c in D. SoxB1b transcripts are detected in the neural plate (arrow) as it is overgrown by epidermal ectoderm. differentiation [41]. 
Figure 3. Embryonic expression of amphioxus SoxB1c. Anterior is to the left. (A) Side view of 9-hour early neurula. SoxB 1 c expression is seen in a patch of neurectoderm near the blastopore (arrow) and in anterior endoderm (arrowhead). (B) Dorsal view of 12-hour neurula, expression in restricted areas of the rostral (arrow) and caudal (arrowhead) neural plate. (C) Side view of 12-hour neurula. SoxB1c transcripts persist in the anterior gut (arrow). (D) Side view of 18-hour late neurula, focused in the plane of the epidermis. Scattered SoxB1c labelled cells are seen in the epidermal ectoderm (arrow). Out of focus, SoxB1c expression has expanded throughout the entire neural tube (double arrowheads) and marks the foregut (arrowhead). (E) Optical cross section through a bisected 18-hour neurula at the level of e in D. Strong expression is seen in the neural tube (arrowhead) and foregut (arrow). (F) Optical cross section through a bisected 18-hour neurula at the approximate the level of $\mathrm{f}$ in $\mathrm{D}$. Arrowheads point to SoxB 1c-positive cells in epidermis. (G) Side view of 24-hour late neurula. Expression in the neural tube (double arrowheads) and foregut (arrowhead) persists, and a band of expression also appears in the hindgut (arrow). (H) Side view of 36-hour late larva. Neural tube (double arrowheads) and hindgut expression have ceased while high levels of transcripts remain in the foregut (arrowhead).

Figure 4. Embryonic expression of amphioxus SoxB2. Anterior is to the left. (A) Side view of 9-hour neurula. SoxB2 transcripts are seen in the caudal-most neurectoderm (arrow) and weakly throughout the ventral mesendoderm (arrowhead). (B) Dorsal view of 9-hour early neurula. SoxB2 transcripts are seen in a small region of neurectoderm bordering the blastopore (arrow). (C) Dorsal view of 12-hour neurula. SoxB2 expression has begun to expand into the anterior neural plate (arrow). (D) Side view of 15-hour neurula. High levels of SoxB2 transcripts are detected throughout the neural tube (arrow). Lower levels are seen in the gut (arrowhead). (E) Optical cross section through a bissected 15-hour neurula at the level of e in D. Strong SoxB2 signal is observed in the neural tube (arrow), while lower levels persist in the gut (arrowhead). By 24 hours, detectable SoxB2 expression has ceased (not shown).

\section{Preservation of amphioxus SoxB1 duplicates with and without obvious subfunctionalization}

The duplication-degeneration-complementation (DDC) model predicts that partitioning of ancestral functions among paralogs after a duplication event will tend to preserve both duplicates, while redundancy will favor the maintenance of just one. In the case of amphioxus SoxB1 paralogs we observe functional partitioning consistent with the DDC model, but also the apparent preservation of two redundant duplicates. Amphioxus SoxB1a is expressed throughout the nascent neural plate but is downregulated before the end of neurulation. It is also never expressed in the endoderm [32]. In contrast, early neurectodermal expression of amphioxus SoxB1c is restricted to rostral and caudal patches (Figure $3 \mathrm{~A}-\mathrm{C})$ until the end of neurulation, when it expands throughout the CNS (Figure 3D). Furthermore, like vertebrate Sox2 and Sox3, amphioxus SoxB1c
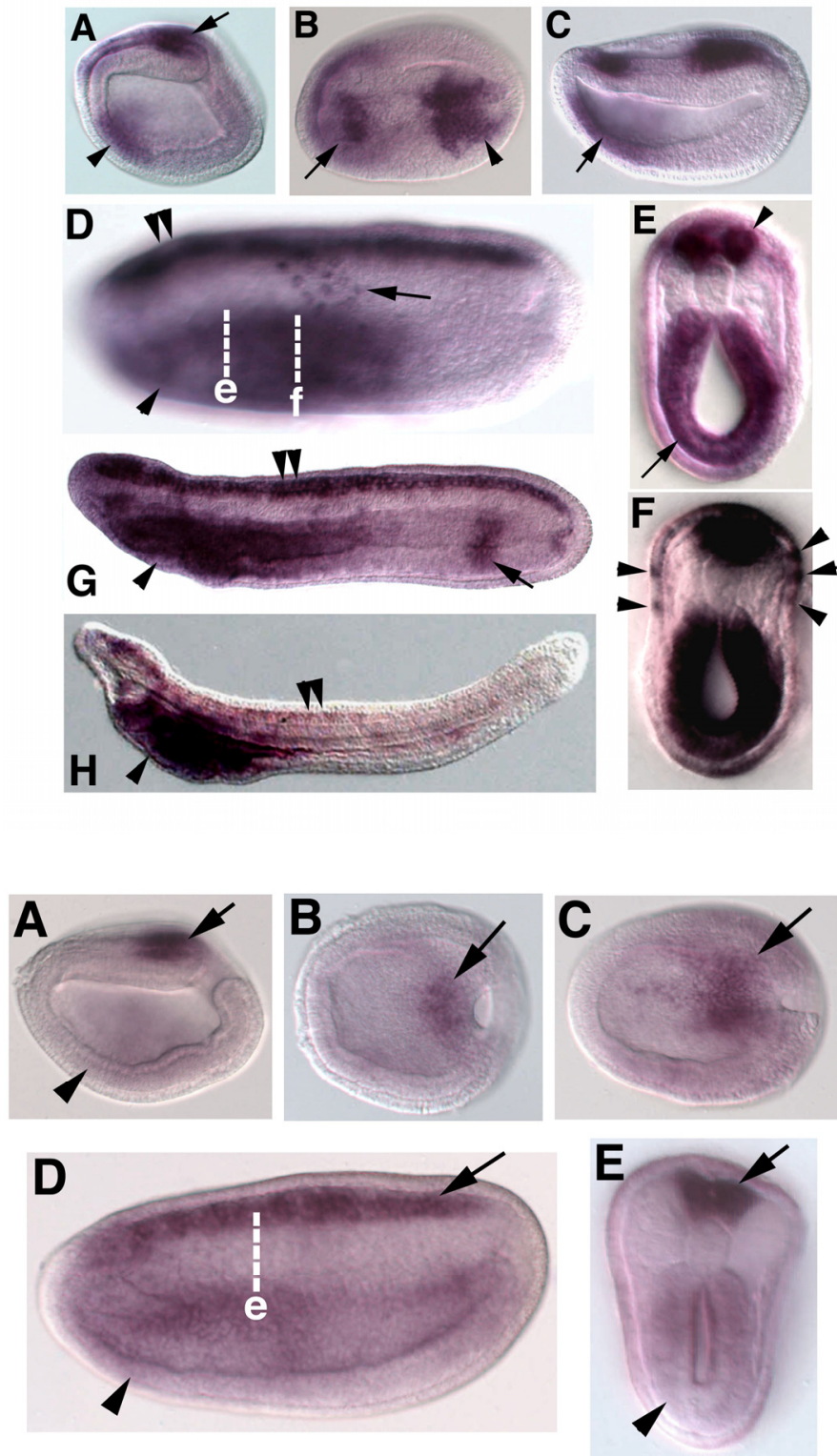

transcripts mark the foregut and hindgut (Figure 3F) and neurogenic ectoderm outside the CNS (discussed below). Thus, amphioxus SoxB1a appears to fulfill the early roles of SoxB1 genes in neural induction, while amphioxus SoxB1c assumes later functions in the developing nervous system and gut. A similar partitioning of early and late functions between amphioxus-specific paralogs is seen in MRF genes [42].

The subfunctionalization of amphioxus SoxB1 genes may explain the divergence of SoxB1a and SoxB1b proteins from other deuterostome SoxB1 homologs, especially in the carboxy-terminal transactivation domain (Figure 1B)[43]. Motifs required for later neural and endodermal functions may have been lost in SoxB1a, resulting in a divergent sequence. Interestingly, this same domain is divergent in Ciona SoxB1, suggesting a similar loss of functionality possibly related to the abbreviated development of urochordates.

In addition to the clear subfunctionalization of 
SoxB1c and SoxB1a, we also observed the redundant expression of SoxB1a and SoxB1b transcripts. Like amphioxus SoxB1a, amphioxus SoxB1b is expressed throughout the nascent neural plate (Figure 2) and is extinguished as SoxB1c is upregulated (Figure 3A-G). The sequence similarity and genomic proximity of SoxB1a and SoxB1b indicate they are the result of a relatively recent duplication event. One explanation for the coexpression of their transcripts is that one duplicate represents a transcribed pseudogene. Alternately, both paralogs may have been preserved to perform different late functions in adult tissues and their redundant early expression reflects responsiveness to the same embryonic enhancers.

Consistent with independent origins for vertebrate and amphioxus SoxB1 paralogs, we noted differences in how the functions of the two sets of paralogs were partitioned during evolution. In vertebrates, all three SoxB1 paralogs are expressed broadly in the early neural plate. Later, vertebrate SoxB1 genes are expressed throughout nervous tissue but in progressively non-overlapping subsets of CNS and PNS neurons [37]. This contrasts with amphioxus in which partitioning of SoxB1 paralogs was largely temporal, with SoxB1a/b being expressed from gastrula until early neurula stages, and SoxB1c being expressed from mid-neurula until larval stages. This difference in subfunctionalization strategy may reflect new roles for vertebrate SoxB1 genes in contributing to the neural complexity of vertebrates.

\section{Conservation of chordate sensory cell specification programs suggested by epidermal expression of amphioxus placode marker homologs}

In the vertebrate PNS, SoxB1 genes are expressed in a subset of neurogenic and non-neurogenic cranial placodes[44], suggestive of cell-type specific functions rather than a general neurogenic role. Anteriorly, vertebrate SoxB1 genes mark the adenohypophysial, olfactory, and lens placodes. Posteriorly, they are expressed in the lateral line, otic and epibranchial placodes, but are excluded from the profundal or trigeminal placodes. Similarly, amphioxus SoxB1c expression is seen in specific subpopulations of general (non-CNS) ectoderm cells, likely presaging formation of two distinct groups of sensory cells. The first population consists of 10-12 cells confined along the anteroposterior axis to the middle third section of lateral epidermis at neurula and early larval stages (Figure 3D,F). This population appears to be a subset of the epidermal sensory cells marked by the more broadly expressed neural markers $H u / E l a v[45,46]$, ERR[47], Trk[48], b-tubulin[49] and Delta[50] and the transcription factor Brn3 (POU IV)[51]. The second group of SoxB1c expressing cells surrounds the forming mouth in larvae (Figure 5A,B). These cells give rise to specialized mechanosensory cells (the oral spine cells) and associated peripheral neurons[15] (Figure 5C).

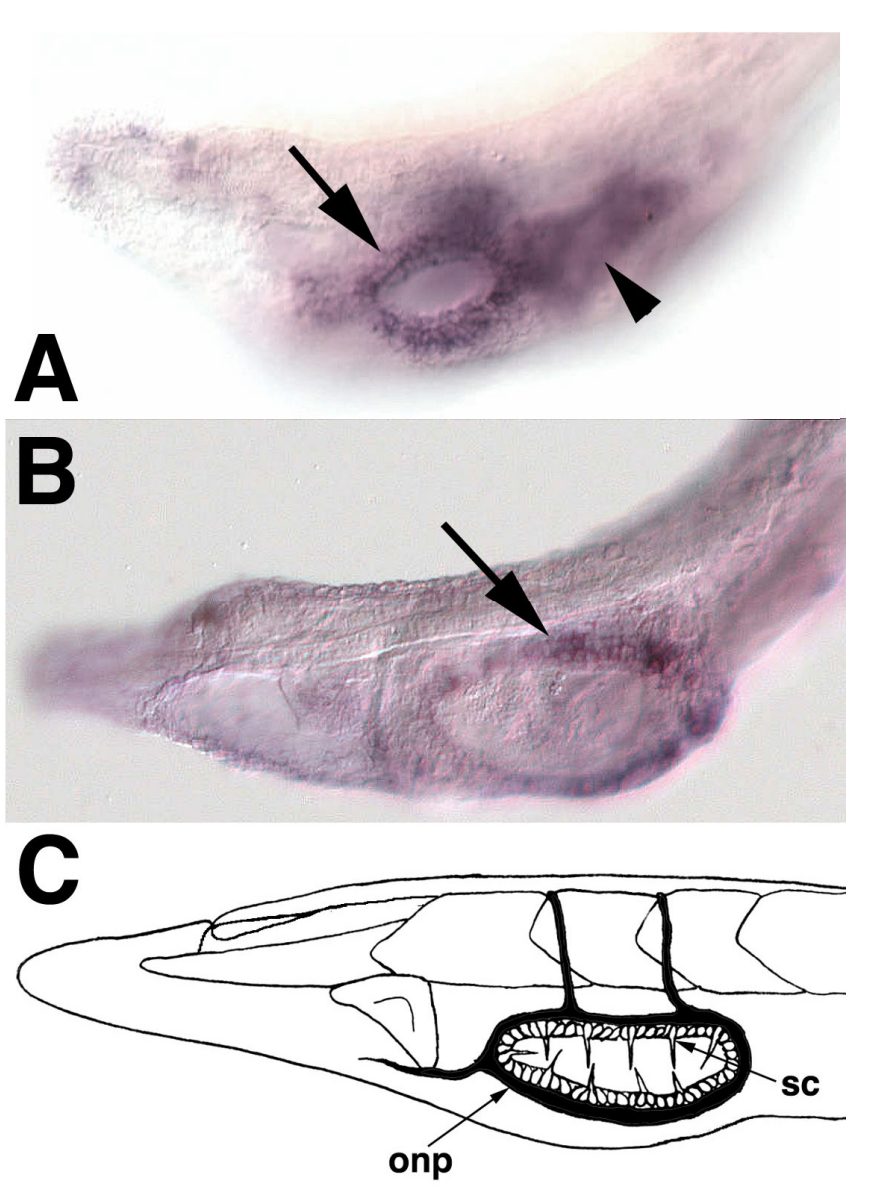

Figure 5. SoxB1c expression in oral ectoderm at early larval stages. Anterior is to the left (A) The head of a 2-day old larva focused in the plane of the epidermis. Expression is apparent in ectoderm surrounding the newly formed mouth (arrow). Out of focus is staining in the underlying pharyngeal endoderm (arrowhead).(B) In 3-day larvae, SoxB1c continues to mark ectoderm around the mouth corresponding to the future location of oral spine cells and neurons. (C) Schematic of oral spine cells, sc, and oral nerve plexus, onp, in 12-14 day larvae modified from Lacalli[63].

Other amphioxus homologs of vertebrate placode markers[44] are expressed in discrete ectodermal domains which generate particular sensory cell subtypes. Vertebrate Pax6 genes are expressed in anterior placodes including the adenohypophyseal, olfactory, lens and trigeminal. In amphioxus, Pax6 marks rostral ectoderm which gives rise to putative chemoreceptors expressing proteins similar in structure to vertebrate olfactory receptors $[9,15,52]$. Vertebrate $M s x$ genes mark the trigeminal, profundal and otic placodes, as well as some lateral line placodes. Amphioxus Msx labels bilateral domains caudal to, and overlapping with, Pax6-positive epidermis which presages formation of putative pressure sensors [15, 53] (the corpuscles of de Quatrefages). Vertebrate Pitx genes collectively label the anteriormost cranial placodes including the adenohypophyseal, olfactory and lens placodes. In amphioxus, Pitx labels the preoral pit and, like SoxB1c, epidermis surrounding 
the mouth which will generate oral spine cells and neurons[8, 54]. The vertebrate homologs of AmphiPOUIV, Brn3a/d, are early markers of posterior placodes including the trigeminal, profundal, lateral line and otic, then later mark the olfactory placode. As mentioned above, amphioxus POUIV/Brn3 appears to mark most epidermal sensory cells in the trunk, the oral epidermis which will form the mouth nerve plexus[51], and a few putative chemosensory cells in the rostrum. Vertebrate Six3 and Six6 are expressed in the anteriormost placodes, including the adenohypophysis and olfactory. Amphioxus Six $3 / 6$ is coexpressed with Pax6 in the putative chemosensory epithelium and preoral pit[55]. Vertebrate Coe/Olf1 genes mark neural derivatives of the olfactory,

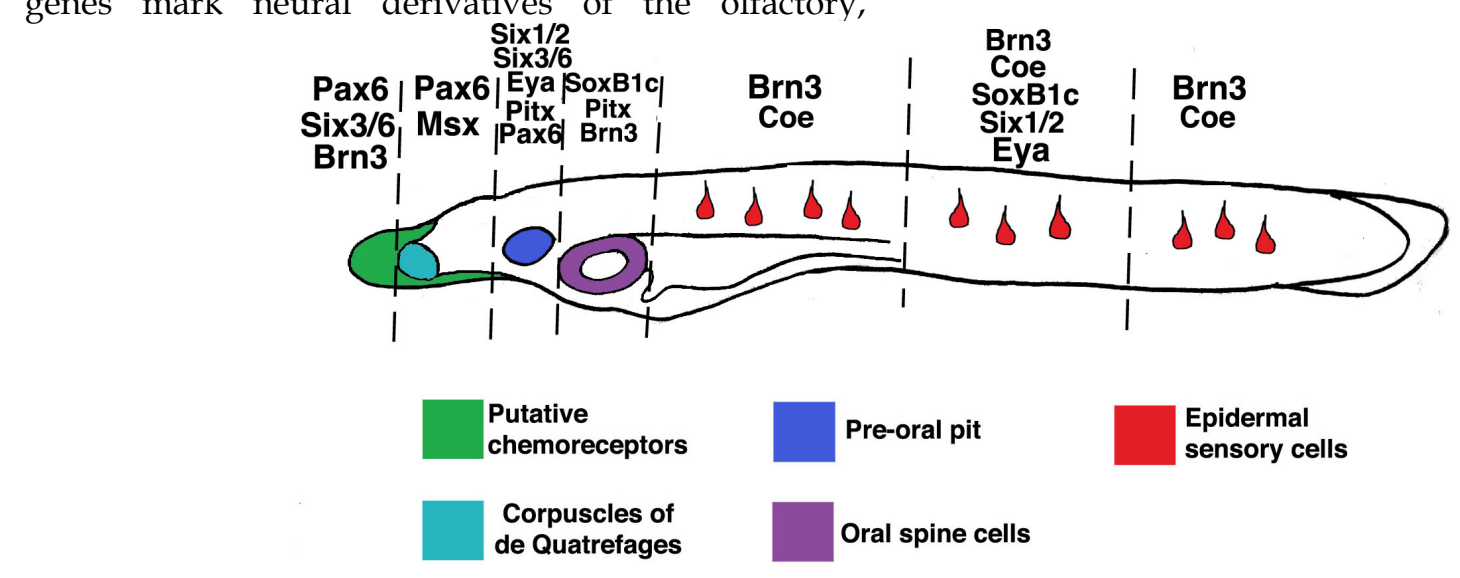

trigeminal and otic placodes [56, 57]. In amphioxus, Coe does not mark the putative olfactory epithelium, though it does mark epidermal sensory cells scattered along the length of neurulae and early larvae. Thus, like their vertebrate cognates, different placode marker homologs label subsets of ectoderm-derived peripheral sensory cells in amphioxus (Figure 6). Furthermore, the rostral-caudal extent of their expression appears partially conserved in both vertebrates and amphioxus. In both groups, Pitx, Pax6, and Six3/6 mark anteriormost cell types, SoxB1 and Brn3 mark posterior cell types, and various combinations of these factors and Msx and Coe are expressed in intervening epidermal cells [44].

Figure 6. Expression of amphioxus placode marker homologs in subsets of epidermal sensory cells. SoxB1c, Pitx, Msx, Pax6, Msx, Six 1/2, Six 3/6, Eya, Coe, and Brn3 homologs mark partially overlapping epidermal domains which give rise to putative sensory cells. Interestingly, the rostro-caudal extent of their expression corresponds roughly to that of their vertebrate cognates in cranial placodes. Though morphologically similar, epidermal sensory cells in the trunk (red) appear deploy different sets of genes. Taken together, these data suggest ancient functions for placode genes in sensory cell specification and a high level of sensory cell diversification in the first chordates.

A notable exception to this general trend is expression of Six1/2 and Eya homologs. In vertebrates, Six1, Six2, and Eya mark virtually all placodes, while in amphioxus, these genes are only expressed in a subset of epidermal sensory cells and the preoral pit [55]. As in vertebrates, urochordate Six1/2 and Eya homologs label broad ectodermal domains rather than individual cells[1, 2]. The expansion of Six1/2 and Eya expression in urochordates and vertebrates relative to amphioxus may reflect expansion of generic placodal properties like the ability to thicken and invaginate. In the first chordates, these properties, and Six/Eya expression, may have been restricted to a pre-oral pit- like organ.

Similar expression of placode marker homologs implies a level of homology between sets of amphioxus epidermal sensory cells and some placodal derivates, though morphological evidence corroborating this is lacking. Most amphioxus epidermal sensory cells are non-descript ciliated mechanosensor-like cells grossly similar to several different vertebrate sensory cells[58, 59]. Others, like the corpuscles of de Quatrefages and oral spine cells, are highly derived and apparently unique to cephalochordates [15]. As previously noted [60], metazoan sensory cell morphology is highly plastic, leading to convergence and rapid divergence and confounding homology assignments. Thus, while placode marker expression in amphioxus and vertebrates suggests the activation of partially conserved sensory cell gene programs, it does not prove strict one-to-one homology between particular amphioxus and vertebrate cells. More accurately, subsets of amphioxus sensory cells likely represent cryptic homologs of particular placode-derived sense cells.

\section{Conserved sensory cell gene programs are likely regulated by different patterning mechanisms in amphioxus and vertebrates.}

In amphioxus, perturbing retinoic acid signaling causes changes in the antero-posterior position of epidermal sensory cells[61]. These changes mirror alterations in Hox gene expression caused by the same treatments, suggesting the antero-posterior patterning of amphioxus epidermal sensory cells may be 
Hox-dependent. Consistent with this, epidermal sensory cells express different complements of Hox genes depending on their axial level, and Hox 1 perturbation mimicks retinoic acid perturbation in the central nervous system[61, 62]. It is possible that an epidermal Hox code activates expression of specific combinations of placode gene homologs which, in turn, drive differentiation of particular sensory cell subtypes at different axial levels in amphioxus. In vertebrates, level-appropriate placode specification does not appear to depend directly on retinoic acid or an epidermal Hox code. Rather, signals emanating from the neural tube and adjacent mesoderm, which are themselves patterned by retinoic acid and Hox genes, induce particular placodes at particular axial levels. Thus, though similar genetic machinery appears to drive sensory cell specification in vertebrates and amphioxus, different patterning mechanisms determine the final position of these cells in the ectoderm. It is likely that changes in the deployment of sensory cell specification programs in the vertebrate lineage involved changes in responsiveness to ectodermal patterning signals.

\section{Diversification of cranial placodes by repeated recruitment of ancient sensory cell gene programs}

Recent phylogenetic[3] and gene expression data from urochordates suggest this group diverged from vertebrates after the evolution of placodes. In both the ascidian Ciona and the larvacean Oikopleura, homologs of the placodal markers Six, Eya, and Pitx label ectodermal domains which undergo localized thickening, invagination, and give rise to sensory cells[1, 2]. The position of these proto-placodes in the larval head is consistent with the existence of two or three composite placodes putatively homologous to the adenohypophsial, olfactory, and otic placodes. In amphioxus, no region of thickened ectoderm appears to combine generic placodal properties with the ability to form sensory cells. However, amphioxus does possess non-neurogenic anterior ectoderm capable of invagination. The amphioxus pre-oral pit is an ectodermal structure formed by invagination. The pre-oral pit also expresses the pan-placodal markers Six1/2 and Eya, and the adenohypophyseal placode markers Pitx, Pax6, and Six $3 / 6$ as well as several adenohypophyseal hormones, leading to speculation that it is homologous to the adenohypophyseal placode[8-11]. Thus, anterior ectoderm displaying generic placodal properties, but incapable of generating sensory cells, was likely present in the first chordates.

Taken together, data from protochordates generally support the model proposed by Schlosser [10] whereby placodes evolved in an amphioxus-like ancestor with scattered epidermal sensory cells and anterior ectoderm capable of invagination (a putative adenohypophyseal placode). The recruitment of sensory cell specification programs to this anterior pre-placodal domain then occurred in the lineage leading to urochordates and vertebrates. Though it is controversial if urochordate and vertebrate placodes are homologous, gene expression and morphology suggest the full complement of vertebrate placodes evolved by partitioning of one or a few proto-placodes. The mechanism by which this may have occurred is unclear. Our data, along with previous studies, suggests placodal diversification arose via recruitment of pre-existing sensory cell specification programs to anterior pre-placodal ectoderm. We speculate that, in the common ancestor of vertebrates and amphioxus, epidermal retinoic acid and Hox patterning mechanisms activated placode marker homologs to specify particular sensory cell fates (Figure 7A). In the vertebrate lineage, these programs lost their dependence on epidermal patterning signals and gained responsiveness to inductive signals in the head. The recruitment of these primitive programs to anterior pre-placodal ectoderm, and their integration into novel gene regulatory networks, drove placodal diversification and the partitioning of proto-placodes (Figure 7B). In cephalochordates, these ancient programs were maintained in the epidermis to specify different sensory cell subtypes. In both lineages, divergence of sensory cell functionality and morphology occurred, obscuring overt evidence of common descent. Though similar expression of homologous genes in chordate sensory cells indicates conservation of some core genetic programming, it is unclear how deeply the gene networks are conserved across the three subphyla. Double-labeling is needed to show precisely which placode gene homologs are coexpressed in which amphioxus sense cells, and functional studies are needed to test if they interact in vertebrate-like gene networks. More rigorous correlation of amphioxus and ascidian placode marker homolog expression with sensory cell position, function, and morphology will also help define the sensory cell complement of the first chordates.

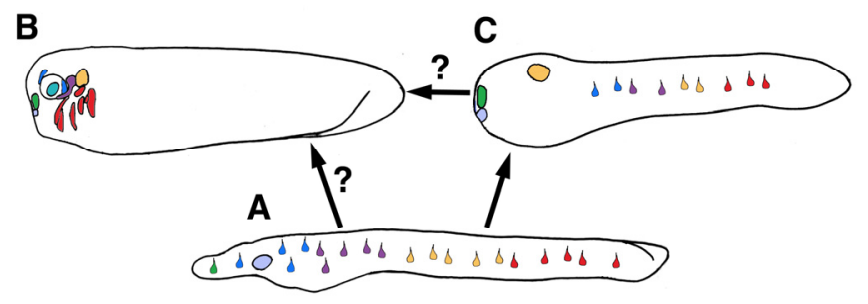

Figure 7. A model for the evolution of placodal diversity. Gene expression in amphioxus suggests placode marker homologs functioned primitively to specify an array of epidermal sensory cell fates in the first chordates (A). In the vertebrates lineage these genes were serially recruited to the anterior ectoderm, driving diversification of a pre-placodal primordium into the full complement of vertebrate placodes. This may have occurred by a loss of responsiveness to retinoic acid patternins mechanisms and a gain of responsiveness to inductive signals in the head. (B) Urochordates may have diverged at an intermediate phase in this process and thus possess some composite placodes putatively homologous to vertebrate cranial placodes, as well as epidermal sensory cells (C). Alternately, urochordate and vertebrate 
placodes may represent parallel structures derived separately from similar sets of epidermal sensory cells.

\section{Acknowledgements}

Phage library was a gift from J. Langeland. Thanks to J.K. Yu for pre-publication access to the amphioxus EST database, J.K. Yu and L.Z. Holland for EST library clones, J. Lawrence for facilities in Tampa, Florida, and the Joint Genome Institute for access to Amphioxus Genome Release v1.0. Funding for this work was provided by NIH grant DE017911 to MBF.

\section{CONFLICT OF INTEREST}

The authors have declared that no conflict of interest exists.

\section{REFERENCES}

1. Bassham S, Postlethwait JH. The evolutionary history of placodes: a molecular genetic investigation of the larvacean urochordate Oikopleura dioica. Development 2005, 132:4259-72.

2. Mazet F, Hutt JA, Milloz J, Millard J, Graham A, Shimeld SM. Molecular evidence from Ciona intestinalis for the evolutionary origin of vertebrate sensory placodes. Dev Biol 2005, 282:494-508.

3. Delsuc F, Brinkmann H, Chourrout D, Philippe H. Tunicates and not cephalochordates are the closest living relatives of vertebrates. Nature 2006, 439:965-8.

4. Blair JE, Hedges SB. Molecular phylogeny and divergence times of deuterostome animals. Mol Biol Evol 2005, 22:2275-84.

5. Garcia-Fernandez J. Amphioxus: a peaceful anchovy fillet to illuminate Chordate Evolution (II). Int J Biol Sci 2006, 2:93-4.

6. Schubert M, Escriva H, Xavier-Neto J, Laudet V. Amphioxus and tunicates as evolutionary model systems. Trends Ecol Evol 2006, 21:269-77.

7. Holland LZ, Laudet V, Schubert M. The chordate amphioxus: an emerging model organism for developmental biology. Cell Mol Life Sci 2004, 61:2290-308.

8. Boorman CJ, Shimeld SM. Pitx homeobox genes in Ciona and amphioxus show left-right asymmetry is a conserved chordate character and define the ascidian adenohypophysis. Evol Dev 2002, 4:354-65.

9. Glardon S, Holland LZ, Gehring WJ, Holland ND. Isolation and developmental expression of the amphioxus Pax-6 gene (AmphiPax-6): insights into eye and photoreceptor evolution. Development 1998, 125:2701-2710.

10. Schlosser G. Evolutionary origins of vertebrate placodes: insights from developmental studies and from comparisons with other deuterostomes. J Exp Zoolog B Mol Dev Evol 2005, 304:347-99.

11. Holland LZ, Holland ND. Evolution of neural crest and placodes: amphioxus as a model for the ancestral vertebrate? Journal of Anatomy 2001, 199:85-98.

12. Gans C, Northcutt RG. Neural Crest and the Origin of Vertebrates - a New Head. Science 1983, 220:268-274.

13. Northcutt RG, Gans C. The genesis of neural crest and epidermal placodes: a reinterpretation of vertebrate origins. Q Rev Biol 1983, 58:1-28.

14. Northcutt RG. The new head hypothesis revisited. Journal of Experimental Zoology Part B-Molecular and Developmental Evolution 2005, 304B:274-297.

15. Lacalli TC. Sensory systems in amphioxus: a window on the ancestral chordate condition. Brain Behav Evol 2004, 64:148-62.

16. Bowles J, Schepers G, Koopman P. Phylogeny of the SOX family of developmental transcription factors based on sequence and structural indicators. Dev Biol 2000, 227:239-55.

17. Kishi M, Mizuseki K, Sasai N, Yamazaki H, Shiota K, Nakanishi $\mathrm{S}$, Sasai Y. Requirement of Sox2-mediated signaling for differentiation of early Xenopus neuroectoderm. Development
2000, 127:791-800.

18. Sandberg M, Kallstrom M, Muhr J. Sox21 promotes the progression of vertebrate neurogenesis. Nat Neurosci 2005, 8:995-1001.

19. Kan L, Israsena N, Zhang Z, Hu M, Zhao LR, Jalali A, Sahni V, Kessler JA. Sox1 acts through multiple independent pathways to promote neurogenesis. Dev Biol 2004, 269:580-94.

20. Groves AK, Bronner-Fraser M. Competence, specification and commitment in otic placode induction. Development 2000, 127:3489-99.

21. Abu-Elmagd M, Ishii $Y$, Cheung M, Rex M, Le Rouedec D, Scotting PJ. cSox3 expression and neurogenesis in the epibranchial placodes. Dev Biol 2001, 237:258-69.

22. Ishii $Y$, Abu-Elmagd M, Scotting PJ. Sox3 expression defines a common primordium for the epibranchial placodes in chick. Dev Biol 2001, 236:344-53.

23. Schlosser G, Ahrens K. Molecular anatomy of placode development in Xenopus laevis. Dev Biol 2004, 271:439-66.

24. Koster RW, Kuhnlein RP, Wittbrodt J. Ectopic Sox3 activity elicits sensory placode formation. Mech Dev 2000, 95:175-87.

25. Kamachi Y, Uchikawa M, Collignon J, Lovell-Badge R, Kondoh $\mathrm{H}$. Involvement of Sox1, 2 and 3 in the early and subsequent molecular events of lens induction. Development 1998, 125:2521-32.

26. Holland ND, Panganiban G, Henyey EL, Holland LZ. Sequence and developmental expression of AmphiDII, an amphioxus Distalless gene transcribed in the ectoderm, epidermis and nervous system: Insights into evolution of craniate forebrain and neural crest. Development 1996, 122:2911-2920.

27. Thompson JD, Gibson TJ, Plewniak F, Jeanmougin F, Higgins DG. The CLUSTAL_X windows interface: flexible strategies for multiple sequence alignment aided by quality analysis tools. Nucleic Acids Research 1997, 25:4876-4882.

28. Saitou N, Nei M. The Neighbor-Joining method- a new method for reconstructing phylogenetic trees. Molecular Biology and Evolution 1987, 4:406-425.

29. Perriere G, Gouy M. WWW-query: An on-line retrieval system for biological sequence banks. Biochimie 1996, 78:364-369.

30. Schmidt HA, Strimmer K, Vingron $M$, von Haeseler A. TREE-PUZZLE: maximum likelihood phylogenetic analysis using quartets and parallel computing. Bioinformatics 2002, 18:502-4.

31. Meulemans D, Bronner-Fraser M. Amphioxus and lamprey AP-2 genes: implications for neural crest evolution and migration patterns. Development 2002, 129:4953-4962.

32. Holland LZ, Schubert M, Holland ND, Neuman T. Evolutionary conservation of the presumptive neural plate markers AmphiSox1/2/3 and AmphiNeurogenin in the invertebrate chordate amphioxus. Dev Biol 2000, 226:18-33.

33. Minguillon C, Ferrier DE, Cebrian C, Garcia-Fernandez J. Gene duplications in the prototypical cephalochordate amphioxus. Gene 2002, 287:121-8.

34. Soriano NS, Russell S. The Drosophila SOX-domain protein Dichaete is required for the development of the central nervous system midline. Development 1998, 125:3989-96.

35. Buescher M, Hing FS, Chia W. Formation of neuroblasts in the embryonic central nervous system of Drosophila melanogaster is controlled by SoxNeuro. Development 2002, 129:4193-203.

36. Overton PM, Meadows LA, Urban J, Russell S. Evidence for differential and redundant function of the Sox genes Dichaete and SoxN during CNS development in Drosophila. Development 2002, 129:4219-28.

37. Uchikawa M, Kamachi Y, Kondoh H. Two distinct subgroups of Group B Sox genes for transcriptional activators and repressors: their expression during embryonic organogenesis of the chicken. Mech Dev 1999, 84:103-20.

38. Ishii Y, Rex M, Scotting PJ, Yasugi S. Region-specific expression 
of chicken Sox2 in the developing gut and lung epithelium: regulation by epithelial-mesenchymal interactions. Dev Dyn 1998, 213:464-75.

39. Wood HB, Episkopou V. Comparative expression of the mouse Sox1, Sox2 and Sox3 genes from pre-gastrulation to early somite stages. Mech Dev 1999, 86:197-201.

40. Chalmers AD, Slack JM, Beck CW. Regional gene expression in the epithelia of the Xenopus tadpole gut. Mech Dev 2000, 96:125-8.

41. Sanchez-Soriano N, Russell S. Regulatory mutations of the Drosophila Sox gene Dichaete reveal new functions in embryonic brain and hindgut development. Dev Biol 2000, 220:307-21.

42. Schubert M, Meulemans D, Bronner-Fraser M, Holland LZ, Holland ND. Differential mesodermal expression of two amphioxus MyoD family members (AmphiMRF1 and AmphiMRF2). Gene Expression Patterns 2003, 3:199-202.

43. Kamachi Y, Cheah KS, Kondoh H. Mechanism of regulatory target selection by the SOX high-mobility-group domain proteins as revealed by comparison of SOX1/2/3 and SOX9. Mol Cell Biol 1999, 19:107-20.

44. Schlosser G. Induction and specification of cranial placodes. Dev Biol 2006, 294:303-51.

45. Benito-Gutierrez E, Illas M, Comella JX, Garcia-Fernandez J. Outlining the nascent nervous system of Branchiostoma floridae (amphioxus) by the pan-neural marker AmphiElav. Brain Res Bull 2005, 66:518-21.

46. Satoh G, Wang Y, Zhang PJ, Satoh N. Early development of amphioxus nervous system with special reference to segmental cell organization and putative sensory cell precursors: A study based on the expression of pan- neuronal marker gene $\mathrm{Hu} /$ elav. Journal of Experimental Zoology 2001, 291:354-364.

47. Bardet PL, Schubert M, Horard B, Holland LZ, Laudet V, Holland ND, Vanacker JM. Expression of estrogen-receptor related receptors in amphioxus and zebrafish: implications for the evolution of posterior brain segmentation at the invertebrate-to-vertebrate transition. Evol Dev 2005, 7:223-33.

48. Benito-Gutierrez E, Nake C, Llovera M, Comella JX, Garcia-Fernandez J. The single AmphiTrk receptor highlights increased complexity of neurotrophin signalling in vertebrates and suggests an early role in developing sensory neuroepidermal cells. Development 2005, 132:2191-202.

49. Yasui K, Tabata S, Ueki T, Uemura M, Zhang SC. Early development of the peripheral nervous system in a lancelet species. J Comp Neurol 1998, 393:415-25.

50. Rasmussen SL, Holland LZ, Schubert M, Beaster-Jones L, Holland ND. Amphioxus AmphiDelta: evolution of delta protein structure, segmentation, and neurogenesis. Genesis 2007, 45:113-122.

51. Candiani S, Oliveri D, Parodi M, Bertini E, Pestarino M. Expression of AmphiPOU-IV in the developing neural tube and epidermal sensory neural precursors in amphioxus supports a conserved role of class IV POU genes in the sensory cells development. Dev Genes Evol 2006, 216:623-33.

52. Satoh G. Characterization of novel GPCR gene coding locus in amphioxus genome: gene structure, expression, and phylogenetic analysis with implications for its involvement in chemoreception. Genesis 2005, 41:47-57.

53. Sharman AC, Shimeld SM, Holland PWH. An amphioxus Msx gene expressed predominantly in the dorsal neural tube. Development Genes and Evolution 1999, 209:260-263.

54. Yasui K, Zhang SC, Uemura M, Saiga H. Left-right asymmetric expression of BbPtx, a Ptx-related gene, in a lancelet species and the developmental left-sidedness in deuterostomes. Development 2000, 127:187-195.

55. Kozmik Z, Holland ND, Kreslova J, Oliveri D, Schubert M, Jonasova K, Holland LZ, Pestarino M, Benes V, Candiani S.
Pax-Six-Eya-Dach network during amphioxus development: Conservation in vitro but context specificity in vivo. Dev Biol 2007; 306(1):143-59.

56. Bally-Cuif L, Dubois L, Vincent A. Molecular cloning of Zcoe2, the zebrafish homolog of Xenopus Xcoe2 and mouse EBF-2, and its expression during primary neurogenesis. Mech Dev 1998, 77:85-90.

57. Dubois L, Bally-Cuif L, Crozatier M, Moreau J, Paquereau L, Vincent A. XCoe2, a transcription factor of the Col/Olf-1/EBF family involved in the specification of primary neurons in Xenopus. Curr Biol 1998, 8:199-209.

58. Holland ND, Yu JK. Epidermal receptor development and sensory pathways in vitally stained amphioxus (Branchiostoma floridae). Acta Zoologica 2002, 83:309-319.

59. Stokes MD, Holland ND. Embryos and Larvae of a Lancelet, Branchiostoma-Floridae, from Hatching through Metamorphosis - Growth in the Laboratory and External Morphology. Acta Zoologica 1995, 76:105-120.

60. Holland LZ. Non-neural ectoderm is really neural: evolution of developmental patterning mechanisms in the non-neural ectoderm of chordates and the problem of sensory cell homologies. J Exp Zoolog B Mol Dev Evol 2005, 304:304-23.

61. Schubert M, Holland ND, Escriva H, Holland LZ, Laudet V. Retinoic acid influences anteroposterior positioning of epidermal sensory neurons and their gene expression in a developing chordate (amphioxus). Proc Natl Acad Sci U S A 2004, 101:10320-5.

62. Schubert M, Holland ND, Laudet V, Holland LZ. A retinoic acid-Hox hierarchy controls both anterior/posterior patterning and neuronal specification in the developing central nervous system of the cephalochordate amphioxus. Dev Biol 2006, 296:190-202.

63. Lacalli TC, Gilmour THJ, Kelly SJ. The oral nerve plexus in amphioxus larvae: function, cell types and phylogenetic significance. Proceedings of the Royal Society of London Series B-Biological Sciences 1999, 266:1461-1470. 